\title{
REVIEW ON THE DEVELOPMENT OF ASSISTED ENDOSCOPIC SURGERY ROBOT TECHNOLOGY
}

\author{
Wang Yan ${ }^{1}$, Hu Tianyu ${ }^{2}$, Zhao Zhonghua ${ }^{3}$ \\ ${ }^{I}$ Mechanical Engineering, Shanghai University of Engineering Science, Shang Hai, China \\ ${ }^{2}$ Mechanical Engineering, Shanghai University of Engineering Science, Shang Hai, China \\ ${ }^{3}$ Engineering Training Center, Shanghai University of Engineering Science, Shang Hai, China
}

\begin{abstract}
Minimally Invasive surgery is an improvement on traditional open surgery. Endoscopy is a technique that can clearly observe the lesions and tissue structure of patients by means of the medical instruments introduced into the body through channels, and facilitate the doctors to deal with the lesions and cut the tissues[1].The application of the assisted endoscope surgery robot in the operation has changed the operation mode fundamentally. The robot supported by the endoscope in the minimally invasive surgery has become one of the hot spots in the international robot research. In this paper, we will make a review of the assisted endoscope surgery robot, hoping to provide convenience for the related researchers.
\end{abstract}

Keywords: - Minimally Invasive surgery, surgery robot, endoscopeassisted -***

\section{A BRIEF HISTORY OF THE DEVELOPMENT}

\section{OF ASSISTED ENDOSCOPIC SURGERY ROBOT}

During the use of endoscope, a medical worker is needed to help support and move it to provide a clear and stable picture for the surgeon. The medical staff who assist endoscope not only need long professional training but also need to keep a high degree of silence with the surgeon so as to avoid interference in the process of operation due to movement errors. The assisted endoscope robot was developed as an opportunity and developed rapidly and steadily in modern medicine treatment.

In the mid-1990s, Computer Motion Company successfully developed the first assisted minimally invasive surgery robot system AESOP-1000 in the world. Which opened the era of assisted endoscope surgery robot. In the same period, Germany developed a passive Tiska endoar system.The system can avoid the effect of additional mechanical force on the incision of the patient and make the endoscope move around this certain point of the patient's incision. The movement is controlled by the doctor through the pedal.In 1995, HISAR system was mainly used for the mechanical arm of endoscope. The structure is designed, and the arm is mounted in the upper space of the operating room to make the endoscope to achieve an ideal posture during the operation.Thus effectively avoiding secondary injury to the patient's incision.In 1998, The Armstrong Medical Centre in the UK has developed a Endoassist robotic assisted endoscope operating system. The head controller can be turned on or off by the switch on the pedal. The system can control the movement of the endoscope according to the direction of the doctor's head motion to obtain the required surgical field of vision.

The first assisted endoscope surgery robot system was commercialized as Computer Motion's AESOP robot[2]. As a representative of commercialization, it can control the movement of endoscope by voice. In 1995, the company developed the ZEUS robot system. The system can be used in all kinds of minimally invasive thoracoabdominal surgery, and even in the correction of cardiac vascular malformation with high coefficient of difficulty. It can use voice or pedal to control the movement of other additional medical devices such as endoscopes[3-6]. At the beginning of this century, the United States Instuitive Surgical has developed the most famous Da Vinci surgical robot system. The end of the manipulator is based on a structure that can realize the telecentric fixed point motion and can realize remote control. Doctors can flexibly control the position and movement of endoscope by using pedals and hands in the main console. The system has been successfully commercialized and is the most widely used assistant endoscope surgery robot[7-8]. According to Reportlinker statistics and analysis, the global market value of robots for medical and health purposes is rising rapidly. With an estimated value of between 2014 and 2019 years, the consistent interest rates can reach $7 \%$. In the Asia-Pacific region, the growth rate is even more obvious[9]. The popularization of minimally invasive surgery and the improvement of the functional structure of endoscope also promote the technological innovation of the assisted endoscope surgery robot, which does not simply relay on the large mechanical arm. Try to be small and flexible. With the development of intelligent and flexible robot, the cost of assisted endoscope surgery robot is high. The delay problem exists in remote surgery and the accurate force feedback and 
the real reproduction of $3 \mathrm{D}$ graphics are lacking. The revolution of endoscope and the innovation of driving mode are all in the direction of assistant endoscope operation robot. The further development of technology is expected to completely replace the assistant medical staff for endoscope control.

\section{THE DEVELOPMENT OF ASSISTED ENDOSCOPIC SURGERY ROBOT}

\section{TECHNOLOGY}

The mechanism of small space assisted endoscope and the force feedback function of real tactile play an important role in the development of assisted endoscope surgery robot. Some of the initial clamping endoscopy mechanisms. Although it can achieve the basic motor function required during the operation, but there are many problems such as tremor, the larger space occupied etc. during the operation. The development of $4 \mathrm{~K}$ high-definition technology can not achieve satisfied effect. The aim of the robot is to provide a clearer tissue structure chart for the operation process. The assisted endoscopic surgery robot has developed all kinds of disciplines in combination, and the upsurge of development and research is still high. The endoscopic surgery robots will be introduced as follows.

\subsection{Da Vinci Surgical Robot System}

The Da Vinci surgical robot system was developed by Instuitive surgical in the United States. After being approved by FDA within a short period of time, it has become commercialized and has been used in more than 2 million surgeries worldwide. Da Vinci system mainly includes remote master console (Fig.1), gripping manipulator (Fig. 2 ), endoscope image processing and surgical instruments used in conjunction. The doctor can operate through the remote console. Using the pedals and the main hand to control the manipulator. Three of the arms are used to operate and the other one is used to hold endoscope. The end structure of the arm is mainly based on the parallelogram principle. The mechanical arm of the assistant endoscope can reach the pathological tissue of the patient flexibly and show clearly to the doctor through the image processing system. It can replace the assistant surgeon for the endoscope clamping.

At present, Da Vinci has developed five generation systems. It continuously enhance the core functions of Da Vinci. Trying to improve the flexibility of the manipulator and increase the motion space at the end of the arm. At the same time, improve and strengthen the imaging system and increase the operation function. Add compatibility and extension space[10-11]. In the actual operation process, we can't completely rely on the robot to perform the operation. All we need is the holding of the Da Vinci high-definition 3D image technology and the increasing clinical perception function instead of the assistant for the endoscope. The cost of its use and maintenance is high. There are many difficulties in its popularizing. The function of force feedback needs to be further enhanced and the defects of time delay need to be overcome in remote control. With the support of powerful technology, Da Vinci surgical robot will be able to play a greater role in the medical field.

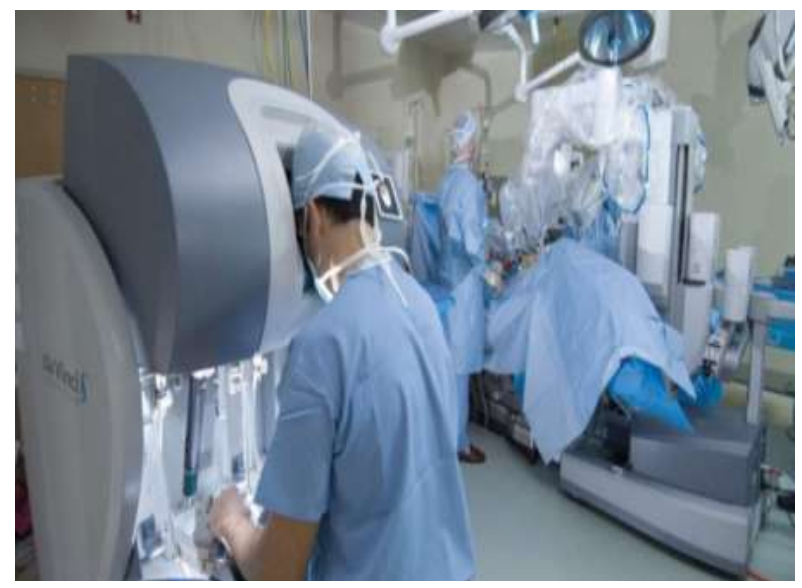

Fig 1

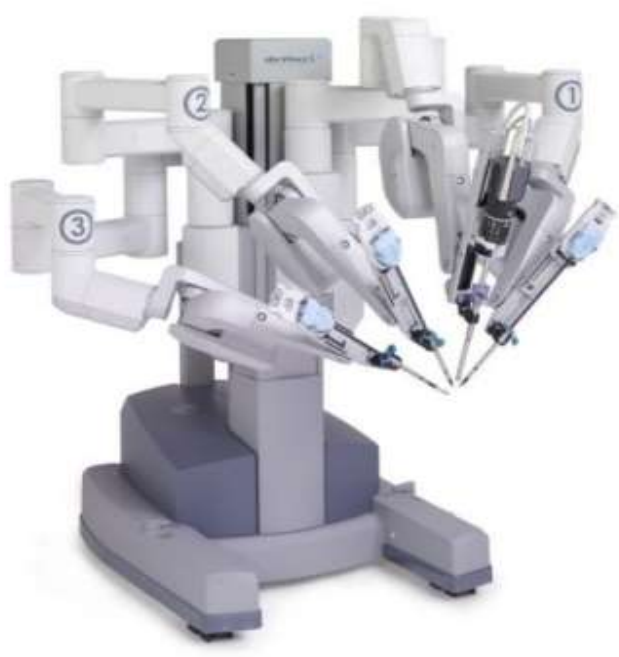

Fig 2

\subsection{Free Hand Surgical Robot System}

Free Hands (Fig. 3) is compact, small and easy to install. There is a control box on the bottom (Fig. 4) fixed on one side of the operating bed. The supporting arm part is passive and depend on the doctor's hand to adjust it to the general required position during the operation. The rotation fixation method is used in the joint which is simple and convenient to operate. At the end of Free Hand, two curved chute are used for motion, which is a kind of scalable structure. The end motion can be controlled by head-mounted controller, and RCM(Remote Centre-of-Motion) can be realized with pedals at the same time. The operation robot is cheap and easy to use and install. However, many of them are manually adjusted, the accuracy is not high. The workload of doctors will be increased during the operation, and the friendly man-machine interaction can not be carried out. 


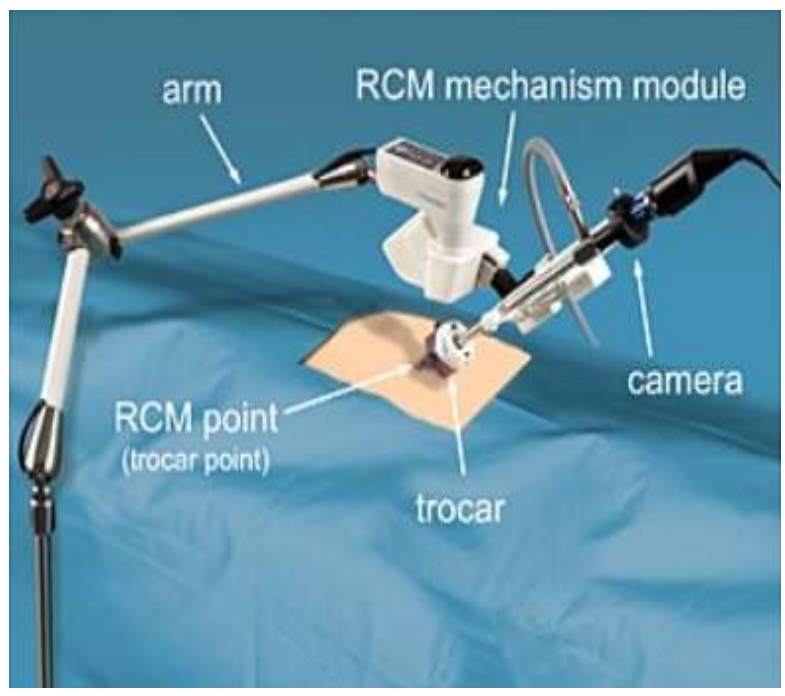

Fig 3

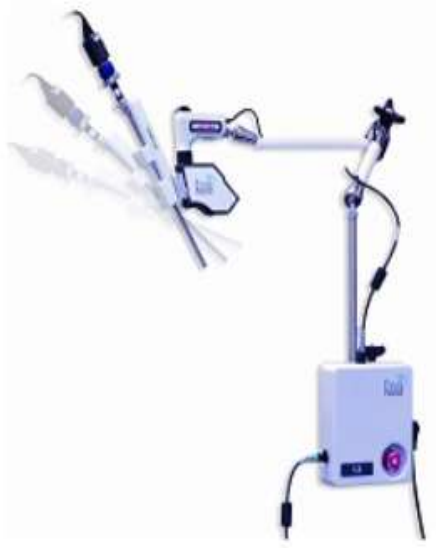

Fig 4

\subsection{SPORT Surgical Robot System}

SPORT (Fig. 5) has only one robotic arm compared to the Da Vinci surgical robot system. It is a relatively simple structure and occupy a small amount of space. The main console is equipped with a high-definition display device. SPORT surgical robot system can install a variety of medical equipment. Its execution workstation also has a self-cleaning lens endoscope device and can achieve singlechannel multifunctional operation. At present, minimally invasive laparoscopic surgery is also developing towards the natural channel. It is necessary to implement single-channel multi-operation surgical instruments. The cost of SPORT is lower than that of Da Vinci robot[12].

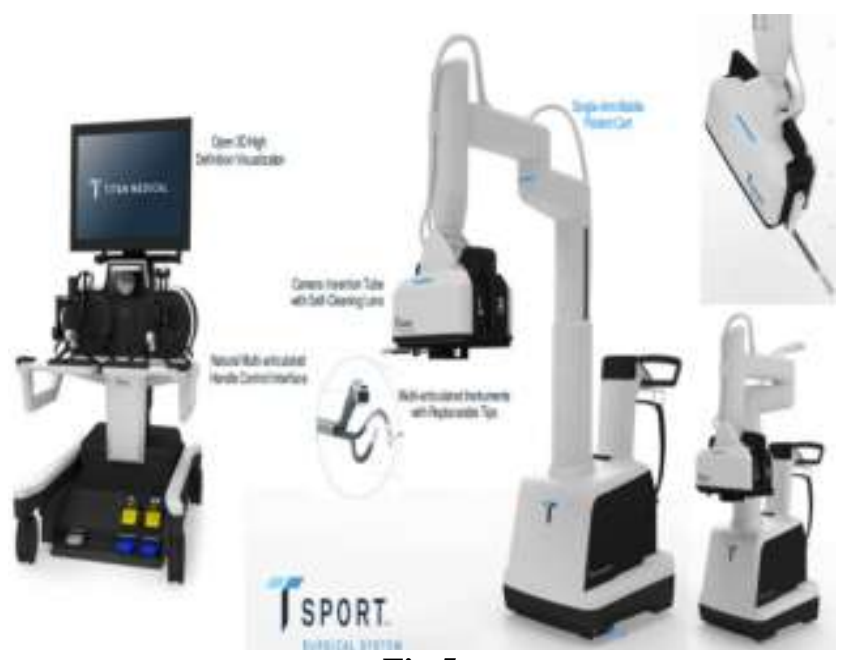

Fig 5

\subsection{Telelap ALF-X Surgical Robot System}

The invention of the Telelap ALF-X robot ( Fig.6 ) is similiar to the Da Vinci in shape.It also has four mechanical arms and capable of working through a remote control workbench. The assisted endoscopic surgery robot system can track the eye of a doctor and control the movement of the endoscope according to the doctor's vision during the operation. In this way, endoscope can be used as the doctor's "eye" in the operation. The system measure the force applied to biological tissue by means of sensors. It's sensitivity can reach $0.35 \mathrm{~N}$.The operative time was significantly shortened during cholecystectomy in animals[13].

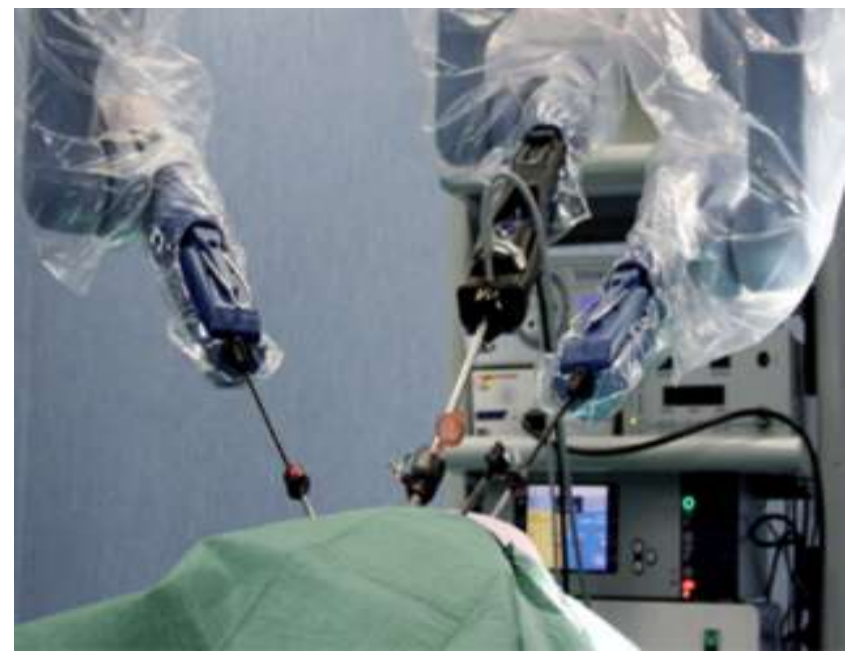

Fig 6

\subsection{Endo Assist Surgical Robot System}

The Endo Assist surgical robot system (Fig. 7) was developed by the UK's Armstrong Medical Centre to replace hand-held endoscopes during minimally invasive surgery. Before the operation, it is necessary to manually align the motion center of the robot with the patient's incision. The motion of the endoscope is controlled by the doctor's head controller, which changes the command according to the turning direction, and the switch of the controller is set on 
the pedal. The control button can be turned off and the doctor's head can rotate freely[14]. This kind of control is common. The doctor's head movement is easy to produce fatigue and the head load will bring physical pressure to the attending doctor. The operation robot system occupies less space in the doctor's operable range. The gripping structure at the end of the system is relatively simple. It is easy to produce tremors and can not provide a clear and stable image.

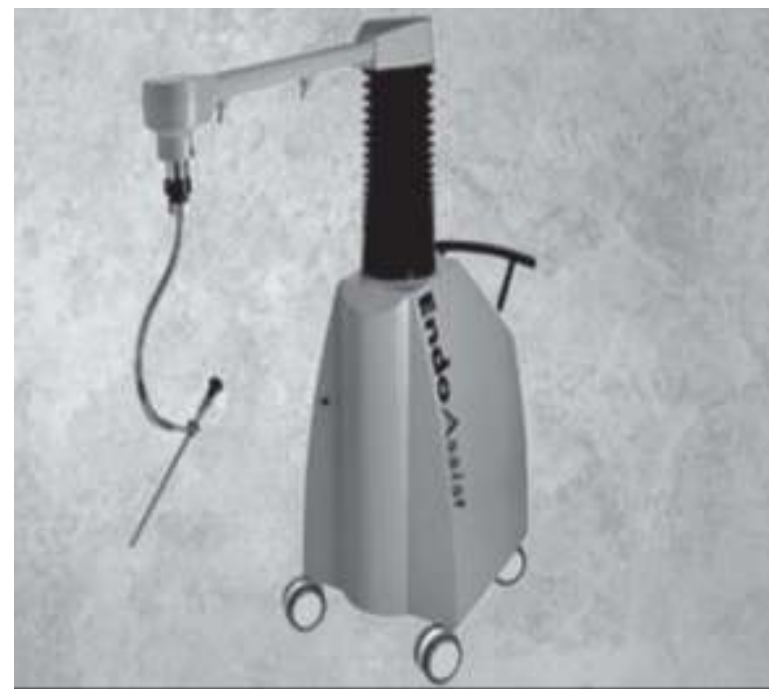

Fig 7

\section{THE FUTURE PROSPECT OF ASSISTED ENDOSCOPIC SURGERY ROBOT}

The generation and development of medical robot is the basis of scientific and technological progress and present human civilization. The assisted endoscope surgery robot is a transition from the robot to complete the operation process in place of the doctor. The assisted endoscope surgery robot can replace the medical assistant to carry on the clinical work. The assisted endoscope surgery robot make the perception, decision, implementation and interaction in combination. The following areas still need to be studied in depth:(1)Enhance the force and torque feedback ability of the assisted endoscope robot. The sensitivity of the Telelap ALF-X robot in force feedback can reach $0.35 \mathrm{~N}$, but it does not completely reach the reality of direct contact. The accuracy of force feedback has a direct impact on the quality of surgery to some extent. (2)Small occupation space. Yuki Kobayashi et al.[15] have studied a small space assisted endoscope surgery robot to produce a high intensity robotic arm. The manipulator is designed to be six degrees of freedom. It uses the active regulating casing and clamping ports. The end motion takes another degree of freedom. (3)Remote manipulation in complex environments. Andreoni C et al.[16] Research on remote control surgery ZEUS has implemented transatlantic surgery. It allowed doctors to operate in a more comfortable environment. The case can effectively relieve the pressure and fatigue caused by the operation. At present, there is the defect of time delay in remote operation. The time delay is about 2-3 seconds, which has a great influence in the actual operation process. (4) Visual automatic guidance control technology. Most of the methods of controlling the movement of endoscope are using head-mounted controller or pedal. The endoscope, as a doctor's "eye", can adjust the movement according to the line of sight in order to play a better role in safety. (5)Reliability of flexible Robot. S. Liu et al.[17] try to use new transmission methods, such as wire drive, to realize assistant endoscope hand. The safety and reliability are necessary for the medical robot. (6) Low cost and high quality. Nowadays, the maintenance cost of the assisted endoscope surgery robot is the main factor that hinders its popularization. The Asia-Pacific region has purchased more medical robotics. The robot system is in the rapid development stage and the assistant endoscope surgery robot is expected to completely replace the medical assistant in the future. Low cost will also promote the popularization and use of the assistant endoscope surgery robot.

\section{CONCLUSION}

Since the emergence of the assisted endoscope surgery robot in the 1990s, the robot has been used more and more widely. It is shown the powerful potential of the robot. Due to the medical aid workers are prone to fatigue during the operation. Assisted endoscope surgery robots can overcome this defect. Under the guidance of the major commercial minimally invasive surgical robots, it is believed that assisted endoscope surgery robots will be more intelligent and exquisite. High precision and friendly interactive will be achieved to make great contributions to the medical service of mankind.

\section{REFERENCES}

[1] FU Yili, PAN Bo, WANG Shuguo. Research on Robot Assisted Endoscope Operating System [J]. Robot Technology and Application, 2006(01):42-47.

[2] TANG Can, WANG Tianmiao, CHOU Wusheng, et al. Design and realization of robot control system for neuro-surgery $[\mathrm{J}]$. Robot, 2004, 26( 6) : 543-547.

[3] Omote K, Feussner H, Ungeheuer A, et al. Selfguided robotic camera control for laparoscopic surgery compared with human camera control[J]. The American journal of surgery, 1999, 177(4): 321-324.

[4] Funda J, Gruben K, Eldridge B, et al. Control and evaluation of a 7-axis surgical robot for laparoscopy[C]//Robotics and Automation,1995. Proceedings., 1995 IEEE International Conference on. IEEE, 1995, 2: 1477-1484.

[5] Taylor R H, Funda J, Eldridge B, et al. A telerobotic assistant for laparoscopic surgery $[\mathrm{J}]$. Engineering in Medicine and Biology Magazine, IEEE, 1995, 14(3): 279-288.

[6] Das H, Ohm T, Boswell C, et al. A Telerobot to Extend the Skill of Microsurgeons[J]. Presence: Teleoperators and Virtual Environments, 1998.

[7] Uneri A, Balicki M A, Handa J, et al. New steadyhand eye robot with micro-force sensing for 
vitreoretinal surgery[C]//Biomedical Robotics and Biomechatronics (Bio Rob), 2010 3rd IEEE RAS and EMBS International Conference on. IEEE, 2010: 814-819.

[8] ZEUS robotic surgical system, Wikipedia, the free encyclopedia. Available from: http://en.wikipedia. org/wiki/ZEUS_robotic_surgical_system

[9] LIANG Kai, CAO Feng, BAI Zhuofu, et al. Detection andprediction of adverse and anomalous events in medicalrobots[C]// Innovative Applications of ArtificialIntelligence, July 14-18, 2013, Bellevue, Washington, USA. California : The AAAI Press, 2013 : 1539-1544.

[10] MOZER P, TROCCAZ J, STOIANOVICI D. Roboticsin urology: past, present, and future [M] / / Atlas of R o-botic Urologic Surgery. New York, USA: Springer, 2011: 3-13.

[11] SHAH K, ABAZA R. Comparison of intraoperative out-comes using the new and old generation da Vinci robot for robot-assisted laparoscopic prostatectomy [ J] . British Journal of Urology International, 2011, 108( 10) : 1642-1645.

[12] NI Ziqiang, WANG Tianmiao, LIU Da. Development of Medical Robot Technology Review[J]. Journal of Mechanical Engineering ,2015,51(13):45-52.

[13] ZHAO Zijian, WANG Fang, CHANG Faliang. Review of Medical Robot Technology in ComputerAided Surgery[J]. Journal of Shandong University (Engineering Edition),2017,47(03):69-78.

[14] WU Litao. Research on Autonomous Micro invasive Surgery Robot Based on Human - Machine Interaction[D]. He'nan University of Technology,2015.

[15] Yuki Kobayashi, Shingo Chiyoda, Kouichi Watabe, Masafumi Okada, and Yoshihiko Nakamura. Small Occupancy Robotic Mechanisms for Endoscopic Surgery[J]. Lecture Notes in Computer Science , $2002,2488: 75-82$

[16] Andreoni C, Araújo M, GattásN, et al. Teleroboticassisted laparoscopic operation performed at a remote site: initial experience[J]. Acta Cirurgica Brasileira, 2004, 19(3): 308-313.

[17] S. Liua, B. Chena, S. Carob, S. Briotb, L. Harewoodc, C. Chena. A cable linkage with remote centre of motion $[\mathrm{J}]$. Mechanism and Machine Theory, 105 (2016) 583-605. 